August, 1997

TAC-1997-028

\title{
Velocity of signal in attractive potential and propagation of light in gravitational field
}

\author{
A.D. Dolgov \\ Teoretisk Astrofysik Center \\ Juliane Maries Vej 30, DK-2100, Copenhagen, Denmark Đ \\ I.B. Khriplovich \\ Budker Institute of Nuclear Physics \\ 630090 Novosibirsk, Russia
}

\begin{abstract}
The propagation of a massless field in attractive and repulsive potentials is considered. It is shown that though the group velocity in such potentials can be larger than one, the wave front propagates with the speed of light. A largerthan-one group velocity leads only to a strong deformation of the wave packet. The results obtained are applied to the light propagation in a gravitational field. An erroneous assertion concerning the last problem, recently made in the literature, is refuted.
\end{abstract}

It is well known that the theory of a free field with a negative mass squared, $m^{2}<0$, is unstable with respect to small perturbations and, moreover, has no ground state. Though it is sometimes argued that a signal in such theories would propagate with a super-luminal velocity, it does not make much sense. In models of spontaneous symmetry breaking the instability introduced by the mass term $m^{2} \phi^{2}$ (with $m^{2}<0$ ) in the potential is "cured" by nonlinear interaction terms, for instance by $\lambda \phi^{4}$. It gives rise to a stable vacuum state and to a positive mass over this vacuum.

It is interesting to consider what would happen to a massless particle propagating through an attractive potential. For a well localized wave packet, with the size which is small in comparison with the characteristic potential range $a$, such a potential is equivalent to a negative mass squared in a finite space region. We study here the problems of stability and of the speed of signal propagation in such a potential. These questions are not of pure methodological interest but refer in particular to the

\footnotetext{
${ }^{1}$ Also: ITEP, Bol. Cheremushkinskaya 25, Moscow 113259, Russia.
} 
physical process of light propagation in an external gravitational background. The Maxwell equations in the empty space can be written as [1-3]:

$$
F_{\mu \lambda ; \nu}^{; \nu}+R_{\mu \lambda \rho \nu} F^{\rho \nu}=0
$$

After diagonalization we obtain from eq. (1) separate equations for the polarization eigenmodes, which describe the propagation of a massless field in gravitational background.

We will model our problem by the Klein-Gordon equation:

$$
-\partial_{t}^{2} \phi=-\Delta \phi+\mu^{2}(x) \phi
$$

Positive $\mu^{2}$ describes a repulsive potential, while $\mu^{2}<0$ describes an attractive one. We consider for simplicity the one-dimensional problem, which in three dimensions corresponds to the forward scattering.

The group velocity in the potential depends upon its sign and is smaller than unity in a repulsive potential for $\omega^{2}>\mu^{2}$ and larger than unity in an attractive potential. This can be easily seen in the eikonal approximation (which is valid if the signal frequency $\omega$ is much larger than both $|\mu|$ and the inverse effective range of the potential, $1 / a\left(\mu^{2} / \omega^{2} \ll 1, \omega a \gg 1\right)$. A wave packet moving in such a potential can be described as

$$
\phi(x, t)=\int_{-\infty}^{\infty} d \omega B\left(\omega-\omega_{0}\right) \exp \left(-i \omega t+i \omega x-\frac{i}{2 \omega} \int_{-\infty}^{x} d x^{\prime} \mu^{2}\left(x^{\prime}\right)\right)
$$

The function $B\left(\omega-\omega_{0}\right)$ is by assumption strongly peaked near the central frequency of the packet $\omega_{0}$. Expanding the exponential near $\omega=\omega_{0}$, we get

$$
\phi(x, t)=e^{-i \omega_{0} t+i \omega_{0} x-i \int_{-\infty}^{x} d x^{\prime} \mu^{2}\left(x^{\prime}\right) / 2 \omega_{0}} A\left[t-x-\frac{1}{2 \omega_{0}^{2}} \int_{-\infty}^{x} d x^{\prime} \mu^{2}\left(x^{\prime}\right)\right]
$$

The function $A$ describes the shape of the wave packet. Its maximum moves with the velocity

$$
v_{g}=1-\mu^{2}(x) /\left(2 \omega_{0}^{2}\right) .
$$

Thus for $\mu^{2}>0$ the group velocity, $v_{g}$, is smaller than the speed of light, while for $\mu^{2}<0$ it is larger than the speed of light. We will show however that the velocity of the front propagation of the signal remains equal to one for both repulsive and attractive potentials. It means in particular that the wave packet is strongly distorted when propagating in "attractive" media, so that its maximum reaches its front. After that the velocity of the propagation of the maximum is equal to that of the front. The shape of the packet becomes very sharp and the usual expressions for the group velocity, in particular (5), which refer to sufficiently smooth packets 
becomes non-applicable. A similar phenomenon takes place in optics in the region of anomalous dispersion (see e.g. [4]).

For a proper approach to the problem of the velocity of signal propagation one has to start with an initial signal which has a sharp front. In other words, the signal should be strictly absent at initial time $t=0$ at distances larger than some $x=x_{0}$. For simplicity we take $x_{0}=0$ so that the wave function does not contain the nonessential phase $\exp \left(i \omega x_{0}\right)$. The region where the potential is essentially nonzero, is assumed to be far to the right from $x=0$. We will expand the solution of eq. (2) in terms of its eigenmodes $\phi_{\omega}(x)$ :

$$
\phi(x, t)=\int_{-\infty}^{\infty} d \omega C(\omega) \phi_{\omega}(x) e^{i \omega t}
$$

We will take $\phi_{\omega}(x)$ in the form which describes an incoming and reflected waves at large negative $x$ and transmitted wave at large positive $x$. Accordingly it has the following asymptotic behavior:

$$
\begin{array}{r}
\phi_{\omega}(x)=e^{i \omega x}+d(\omega) e^{-i \omega x} \quad(\text { for } x \rightarrow-\infty), \\
\phi_{\omega}(x)=f(\omega) e^{i \omega x} \quad(\text { for } x \rightarrow \infty) .
\end{array}
$$

To satisfy the above mentioned condition of absence of the incoming wave at $x>0$ for $t<0$, the coefficient function $C(\omega)$ should be analytic in the upper-half plane of complex $\omega$. Indeed, in this case the contour of integration in $\omega$ can be closed in upper half-plane for $(x-t)>0$ and the corresponding integral vanishes.

In three-dimensional case we are interested in the forward scattering amplitude $f(\omega)$. Its analytical properties follow directly from the analysis of the operator

$$
H=-\Delta+U(r) \equiv-\Delta+\mu^{2}(r),
$$

as is discussed in textbooks on quantum mechanics (see e.g. [5, 6]). The amplitude is analytic in the upper half-plane $\omega$ and for sufficiently strong attractive potentials has poles on the positive imaginary axis which correspond to bound states. In onedimensional case the analytical properties of the functions $d(\omega)$ and $f(\omega)$ are the same (see e.g. [9, 7]), with the only difference that an attractive one-dimensional potential has usually at least one bound state and respectively at least one pole on the upper imaginary axis.

Let us start from the study of the wave front propagation for the repulsive potential. The amplitude of the transmitted wave is given by eq. (可) where the product $C(\omega) f(\omega)$ is analytic in the upper half plane. Thus by the same reason that the incoming wave vanishes for $x>t$, the transmitted wave vanishes in the same interval and the super-luminal propagation is impossible. We came effectively to the known 
statement [8] that the velocity of the wave front is determined by the asymptotics of the refraction index $n(\omega)$ as $\omega \rightarrow \infty$. In turn this asymptotics is obviously governed by the characteristics of the wave equation which are not influenced by the potential. Thus the velocity of the wave front propagation does not exceed the speed of light (in the case considered it is equal to the speed of light), though the group velocity generally differs from unity. The group velocity may be larger than speed of light but the wave front goes with the speed of light.

We are considering here the problem of propagation of signal in attractive potentials or, if in repulsive ones, then well above the barrier. As to the case of the barrier penetration by a signal, strong deformation of the wave packet results here in nontrivial phenomena. However, their discussion is beyond the scope of the present article. A detailed treatment of the problem of sub-barrier propagation of a signal as well as the list of appropriate references, both theoretical and experimental, is presented in ref. [10], where the general expression for the time-delay of the signal for sub-barrier motion has been calculated.

Let us turn now to the case of attractive potential. When the potential is sufficiently weak, the scattering amplitude, as mentioned above, is analytic in the upper half-plane and the problem of signal propagation does not differ at all from the already considered case of repulsive potential. In a strong potential the operator $H=-\Delta+\mu^{2}(x)$ acquires negative eigenvalues, $\omega_{j}^{2}<0$, which correspond to imaginary $\omega_{j}$ and to unstable eigenmodes, $\phi_{\omega} \sim \exp \left(\left|\omega_{j}\right| t\right)$. Small fluctuations in such a potential exponentially grow up and the initial problem with a signal absent for $x>0$ is not so trivial from the physical point of view, as it is above in the stable case. Still we can consider the following mathematically well defined problem, namely the propagation of the signal with sharp front, $\phi(x, 0)=0$ for $x>0$ assuming that the rising fluctuations are absent. We will present the wave function in the same form as above, eq. (6), but with the integration done along the contour which goes over the singularities in the upper half-plane (see Fig. 1). Let us emphasize that this choice of the contour is necessary for the meaningful formulation of the problem, with the signal absent for $x>0$. One can see that again the signal propagates with the speed of light, i.e. $\phi(x, t)=0$ for $x>t$.

It can be seen that the signal propagates without essential deformation till it reaches the region of the potential. Here it excites the unstable modes which also propagate with the speed of light, but exponentially grow behind the wave front, $\phi \sim$ $\exp \left|\omega_{j}(x-t)\right|$. The amplitudes of these unstable modes are equal to the coefficients of decomposition of the incoming wave in terms of the unstable eigenmodes of the operator $H$. There are of course other rising contributions which are not related to the propagating wave. They originate from the unstable fluctuations (noise) in the region of non-vanishing potential.

One can however distinguish experimentally the propagating signal, which may 
carry information, from the unstable exponentially rising noise. Let us assume that the initial signal is a wave packet with a high central frequency $\omega_{0} \gg\left|\omega_{j}\right|$. To carry some information, this signal should be modulated with the frequency $\tilde{\omega}$ which is also much larger than $\left|\omega_{j}\right|$. Of course the noise coming from the unstable fluctuations may reach a detector faster than the signal and at first sight can mimic an acausal propagation. However the detector can distinguish between them. For example the detector may be constructed in such a way that it does not register the slowly varying background. If it is sensitive only to a high frequency signal and if the measuring time is small in comparison with $1 /\left|\omega_{j}\right|$ and large in comparison with $1 / \omega_{0}$ and $1 / \tilde{\omega}$, the detector would react only to causally propagating signal.

Coming back to the problem of the propagation of electromagnetic waves in a gravitational background, we see that neither the "potential" term (proportional to

$R_{\mu \nu \rho \lambda}$ ) nor the terms with the Christoffel symbols implicit in $F_{\mu \nu ; \lambda} ; \lambda$, influence the characteristics of the wave equation and therefore do not change the velocity of the wave front propagation which remains equal to the speed of light. (It sounds funny: "speed of light is equal to the speed of light", but the meaning is clear.) However the radiative corrections to the Maxwell equations change the second-derivative terms and correspondingly do change the characteristics of the wave equation [11, 12]. This can make in particular the velocity of wave front propagation larger than one. The criticism of this conclusion made in ref. [3] is based on the erroneous identification of the signal velocity with the group velocity instead of the wave front one.

Let us mention in conclusion that strong attractive fields indeed exist in nature. We mean the gravitational fields of black holes at $r \sim r_{g}$. The vacuum instability in these fields results in the Hawking radiation.

\section{Acknowledgment.}

This work was supported in part by Danmarks Grundforskningsfond through its funding of the Theoretical Astrophysical Center (TAC). I.Kh. thanks TAC for hospitality and acknowledges the support by the Russian Foundation for Basic Research through grant No. 95-02-04436-a.

\section{Figure caption.}

Integration contour in the complex $\omega$-plane in eq. (6). 


\section{References}

[1] A.S. Eddington, The Mathematical Theory of Relativity (Cambridge University Press, 1957), p. 176.

[2] T.W. Noonan, Ap. J. 341 (1989) 786.

[3] S. Mohanty and A.R. Prasanna, gr-qc/9701009.

[4] M. Born and E. Wolf, Principles of Optics (Pergamon Press, 1968), § 1.3.4.

[5] L.D. Landau and E.M. Lifshits, Quantum Mechanics (Pergamon Press, 1991), $\S 129$.

[6] V. DeAlfaro and T. Regge, Potential Scattering (North Holland, 1965).

[7] M.S. Marinov and B. Segev, J. Phys. A: Math. Gen. 29 (1996) 2839.

[8] M.A. Leontovich, in: L.I. Mandelshtam, Lectures in Optics, Relativity, and Quantum Mechanics (Nauka, Moscow, 1972), p. 308.

[9] V.E. Zakharov, S.V. Manakov, S.P. Novikov, and L.P. Pitaevskii, Theory of Solitons: Method of Inverse Problem (Nauka, Moscow,1980), pp. 23-25.

[10] M.S. Marinov and B. Segev, Phys. Rev. A 55 (1997) 3580.

[11] I.T. Drummond and S.J. Hathrell, Phys. Rev. D 22 (1980) 343.

[12] I.B. Khriplovich, Phys. Lett. B 346 (1995) 251. 